\title{
Entropy on Intuitionistic Fuzzy Sets and Hesitant Fuzzy Sets
}

\author{
Bicheng Yu $\mathbb{D}^{1}{ }^{1}$ Xuejun Zhao, ${ }^{1}$ Mingfa Zheng, ${ }^{1}$ Xiujiu Yuan, ${ }^{1}$ and Bei Hou ${ }^{2}$ \\ ${ }^{1}$ Department of Basic Science, Air Force Engineering University, Xi'an, China \\ ${ }^{2}$ Information and Navigation College, Air Force Engineering University, Xi'an, China \\ Correspondence should be addressed to Bicheng Yu; 2328214244@qq.com
}

Received 31 August 2021; Revised 20 November 2021; Accepted 3 December 2021; Published 7 February 2022

Academic Editor: Ching-Feng Wen

Copyright (C) 2022 Bicheng Yu et al. This is an open access article distributed under the Creative Commons Attribution License, which permits unrestricted use, distribution, and reproduction in any medium, provided the original work is properly cited.

Since the sufficient conditions for the maximum value of the intuitionistic fuzzy entropy are not unified and the hesitant fuzzy entropy cannot be compared when the lengths of the hesitation fuzzy elements are not equal, improved axiomatic definitions of intuitionistic fuzzy entropy and hesitant fuzzy entropy are proposed, and new intuitionistic fuzzy entropy and hesitant fuzzy entropy based on the improved axiomatic definitions are established. This paper defines the fuzzy entropy that satisfies the properties based on the axiomatized definition of fuzzy entropy and, based on the fuzzy entropy, defines new intuitionistic fuzzy entropy and hesitant fuzzy entropy, so that the three are unified in form. The validity and rationality of the proposed intuitionistic fuzzy entropy and hesitant fuzzy entropy are verified by analysis.

\section{Introduction}

The concept of entropy was proposed by Clausius in 1958 and applied to thermodynamics to measure the degree of energy exhaustion in a material system. Entropy is an important concept for measurement uncertainty. Shannon called the uncertainty in the communication process information entropy and constructed a functional information entropy formula. After Zadeh [1] first introduced fuzzy sets in 1965, DeLuca and Termini [2] presented the axioms with which the fuzzy entropy should comply and defined the entropy of fuzzy sets based on Shannon's function. Among the various developments of fuzzy sets theory, the research on intuitionistic fuzzy sets and hesitant fuzzy sets is the most representative. Similarly, intuitionistic fuzzy entropy and hesitant fuzzy entropy have attracted extensive research by scholars.

Intuitionistic fuzzy sets [3-6] were proposed by Atanassov in 1983. Intuitionistic fuzzy sets are an important extension of fuzzy sets, which have a wide range of applications in many fields, such as multiattribute decisionmaking [7-9], pattern recognition [10], and image segmentation [11]. Intuitionistic fuzzy set considers the information of membership, nonmembership, and hesitancy degree. Therefore, it is better than traditional fuzzy sets in dealing with fuzziness and uncertainty and is more flexible and practical. In 1996, Burillo [12] first proposed the concept of intuitionistic fuzzy entropy, gave an axiomatic definition (abbreviated as B-B axiomatic definition), and constructed the calculation formula of intuitionistic fuzzy entropy that satisfies the axiom condition. In 2001, Szmidt [13] extended the definition of information entropy to the intuitionistic fuzzy environment and proposed four constraints different from the B-B axiomatic definition to define the intuitionistic fuzzy entropy (abbreviated as S-K axiomatic definition). In 2006, from the perspective of probability, Hung [14] regarded the three elements as probabilistic measures based on the assumption that " the sum of membership degree, non-membership degree, and hesitation degree of the intuitionistic fuzzy set is always 1 " and accordingly gave a new axiomatic definition (referred to as $\mathrm{H}-\mathrm{Y}$ axiomatic definition). In 2009, Zhang [15] gave a new axiomatic definition of intuitionistic fuzzy entropy based on the distance between intuitionistic fuzzy sets (referred to as Zhang axiomatic definition). The axiomatic definition of intuitionistic fuzzy entropy in literature [12-15] fails to fully reflect the fuzziness of intuitionistic fuzzy sets, especially in terms of the necessary and sufficient conditions for 
restraining the maximum value of intuitionistic fuzzy entropy, which does not meet reality, and fails to objectively describe the maximum or minimum fuzzy degree of the intuitionistic fuzzy set.

The hesitant fuzzy sets [16] were put forward by Torra in 2010 in order to be able to effectively deal with the decisionmaking problem of uncertain information. Compared with fuzzy sets, hesitant fuzzy sets can more effectively solve multiattribute decision-making problems in uncertain environments. In recent years, the research on the entropy measurement of hesitant fuzzy sets has attracted widespread attention from scholars. For example, Wei [17] proposed a series of hesitant fuzzy entropy based on the mean and variance of hesitant fuzzy elements; $\mathrm{Hu}$ [18] studied the hesitant fuzzy entropy based on the similarity of the hesitation fuzzy. Xu [19] discussed the fuzzy entropy and cross entropy in the hesitant fuzzy environment; proposed several measurement formulas about the hesitant fuzzy set; and analyzed the relationship between the hesitant fuzzy entropy, cross entropy, and similarity measurement. Liang [20] constructed a multiattribute decision-making model based on the entropy weight method according to the scoring function and the principle of minimum relative entropy. In fact, the hesitant fuzzy entropy is extended from the concept of fuzzy entropy; that is, the entropy of each membership degree in the hesitation fuzzy element should satisfy the definition of fuzzy entropy. In actual decision-making problems, when the hesitant fuzzy element and its complement are the same, according to some existing hesitant fuzzy entropy formulas, the entropy of the hesitant fuzzy element is always calculated to be 1 , so that the weight of the attribute information is exactly the same. This cannot reflect the preferences of decision makers.

This paper analyzes the defects of the axiomatic definitions of intuitionistic fuzzy entropy and hesitant fuzzy entropy in detail, proposes improved axiomatic definitions of intuitionistic fuzzy entropy and hesitant fuzzy entropy, and constructs a new intuitionistic fuzzy entropy and hesitant fuzzy entropy calculation formula based on the improved axiomatic definition. The fuzzy entropy, intuitionistic fuzzy entropy, and hesitant fuzzy entropy are unified in form.

\section{Preliminaries}

In this section, we briefly review the basic concepts related to fuzzy sets, intuitionistic fuzzy sets, and hesitant fuzzy sets and then list some operations defined on them. The connection between them is also presented.

\subsection{Fuzzy Set}

Definition 1 (see [1]). Let $X=\left\{x_{1}, x_{2}, \ldots, x_{n}\right\}$ be the universe of discourse; then, a fuzzy set $A$ in $X$ is defined as

$$
A=\left\{\left\langle x, \mu_{A}(x)\right\rangle \mid x \in X\right\},
$$

where $\mu_{A}(x): X \longrightarrow[0,1]$ is the membership degree.
Definition 2 (see [1]). For any two fuzzy sets $A$ and $B$ in $X$, the following relations and operations can be defined:

(1) $A(x) \cup B(x)=A(x) \vee B(x), \forall x \in X$

(2) $A(x) \cap B(x)=A(x) \wedge B(x), \forall x \in X$

(3) $A^{c}(x)=1-A(x), \forall x \in X$

\subsection{Intuitionistic Fuzzy Set}

Definition 3 (see [3]). Let $X=\left\{x_{1}, x_{2}, \ldots, x_{n}\right\}$ be the universe of discourse; an intuitionistic fuzzy set (IFS) $A$ in $X$ is defined as

$$
A=\left\{\left\langle x, \mu_{A}(x), v_{A}(x)\right\rangle \mid x \in X\right\},
$$

where $\mu_{A}: X \longrightarrow[0,1], v_{A}: X \longrightarrow[0,1]$ are membership degree and nonmembership degree of the element $x \in X$, respectively, and $\forall x \in X, 0 \leq \mu_{A}(x)+\nu_{A}(x) \leq 1 . \pi_{A}(x)$ is determined by the following equation:

$$
\pi_{A}(x)=1-\mu_{A}(x)-v_{A}(x) .
$$

$\pi_{A}(x)$ is called the hesitancy degree of the element $x \in X$ to the IFS $A . \pi_{A}(x) \in[0,1], \forall x \in X$. Greater $\pi_{A}(x)$ indicates more vagueness on $x$. Obviously, when $\pi_{A}(x)=0, \forall x \in X$, an IFS degenerates into an ordinary fuzzy set.

Definition 4 (see [3]). For any two IFSs $A$ and $B$ in $X$, the following relations and operations can be defined:

(1) $A \leq B \Leftrightarrow \mu_{A}(x) \leq \mu_{B}(x), \nu_{A}(x) \geq \nu_{B}(x)$

(2) $A=B \Leftrightarrow A \leq B, B \leq A$

(3) $A \cap B=\left\{\left\langle x, \min \left(\mu_{A}(x), \mu_{B}(x)\right), \max \left(\nu_{A}(x), v_{B}\right.\right.\right.$ $(x))\rangle \mid x \in X\}$

(4) $A \cup B=\left\{\left\langle x, \max \left(\mu_{A}(x), \mu_{B}(x)\right), \min \left(\nu_{A}(x), v_{B}\right.\right.\right.$ $(x))\rangle \mid x \in X\}$

(5) $A^{c}=\left\{\left\langle x, v_{A}(x), \mu_{A}(x) \mid x \in X\right\rangle\right\}$

\subsection{Hesitation Fuzzy Set}

Definition 5 (see [16]). Let $X=\left\{x_{1}, x_{2}, \ldots, x_{n}\right\}$ be the universe of discourse. A hesitant fuzzy set (HFS) $A$ in $X$ is defined as

$$
A=\left\{\left\langle x_{i}, h_{A}\left(x_{i}\right)\right\rangle \mid x_{i} \in X\right\},
$$

where $h_{A}\left(x_{i}\right)$ is a set of some values in $[0,1]$, which is called the hesitant fuzzy element (HFE), denoting the possible membership degrees of the element $x_{i} \in X$ to the set $A$. In particular, if there is only one value in each $h_{A}\left(x_{i}\right)$ $(i=1,2, \ldots, n)$, then the HFS $A$ degenerates into an ordinary fuzzy set, which indicates that fuzzy sets are a special type of HFSs. HFS $(X)$ is the set of all the HFSs on $X$.

It is assumed that for any HFE $h=\left\{\gamma^{1}, \gamma^{2}, \ldots, \gamma^{l}\right\}$, when $m<n(m, n=1,2, \ldots, l)$, there is $\gamma^{m} \leq \gamma^{n}$, where $\gamma^{\lambda} \in[0,1]$, $\lambda=1,2, \ldots, l$, and $l$ is the length of elements in $h$. 
Definition 6. (see [16]). For any two HFEs $h_{1}$ and $h_{2}$ in $X, \gamma^{1}$ and $\gamma^{2}$ are, respectively, any element in $h_{1}$ and $h_{2}$; the following relations and operations can be defined:
(1) $h_{1}^{c}=\cup_{\gamma^{1} \in h_{1}^{c}}\left\{1-\gamma^{1}\right\}$
(2) $h_{1} \cup h_{2}=\cup_{\gamma^{1} \in h_{1}, \gamma^{2} \in h_{2}} \max \left\{\gamma^{1}, \gamma^{2}\right\}$
(3) $h_{1} \cap h_{2}=\cap_{\gamma^{1} \in h_{1}, \gamma^{2} \in h_{2}} \min \left\{\gamma^{1}, \gamma^{2}\right\}$

2.4. Entropy Measures for Fuzzy Sets. Fuzzy entropy describes the uncertainty of fuzzy sets, which satisfies the following:

(1) If the membership degree of the fuzzy set is 0 or 1 , then the fuzzy entropy of the fuzzy set is 0

(2) If the membership degree of the fuzzy set is 0.5 , then the fuzzy entropy of the fuzzy set is the largest

(3) The closer the membership degree of the fuzzy set is to 0.5 , the larger its fuzzy entropy is; conversely, the further away it is from 0.5, the smaller its fuzzy entropy is

(4) The fuzzy entropy of fuzzy set is equal to the fuzzy entropy of its complement

Definition 7. Let $F(X)$ denote the set of all fuzzy sets over $X$. A mapping $H: F(X) \longrightarrow[0,1]$ is called an fuzzy entropy if it satisfies the following properties:

(1) $\forall x \in X, \mu_{A}(x) \in\{0,1\} \Leftrightarrow H(A)=0$

(2) $\forall x \in X, \mu_{A}(x)=0.5 \Leftrightarrow H(A)=1$

(3) $\forall x \in X, \mu_{A}(x) \leq \mu_{B}(x) \leq 0.5 \quad$ or $\quad \mu_{B}(x) \geq \mu_{A}(x) \geq$ $0.5 \Leftrightarrow H(B) \leq H(A)$

(4) $\forall A \in F(X), H(A)=H\left(A^{c}\right)$

Definition 8. For each $x_{i} \in X, H_{i}(A)$ is defined by

$$
H_{i}(A)=-4\left(\mu_{A}\left(x_{i}\right)-0.5\right)^{2}+1=-4 \mu_{A}^{2}\left(x_{i}\right)+4 \mu_{A}\left(x_{i}\right) .
$$

Then, $H_{i}(A)$ is an uncertainty measure for fuzzy set elements $x_{i}$.

Let us consider the following function:

$$
f(x)=-4 x^{2}+4 x, \quad 0 \leq x \leq 1 .
$$

Since $f(x)=f(1-x)$, we can get that $f(x)$ is symmetric about $x=0.5$.

Then, we have $f^{\prime}(x)=-8 x+4$; when $x \geq 0.5, f^{\prime}(x) \leq 0$, $f(x)$ decreases monotonically with $x$; when $x \leq 0.5$, $f^{\prime}(x) \geq 0, f(x)$ increases monotonously with $x$; when $x=0.5, f(x)$ gets its maximum value $f(x)_{\max }=f(0.5)=1$; when $x=0$ or $1, f(x)$ gets the minimum value $f(x)_{\min }=f(0)=f(1)=0 . f(x)$ is shown in Figure 1 .

From the above discussion, we can get $0 \leq H_{i}(A) \leq 1$.

Definition 9. For each $A \in F(X), H(A)$ is defined by

$$
H(A)=-\frac{1}{n} \sum_{i=1}^{n}\left[-4 \mu_{A}^{2}\left(x_{i}\right)+4 \mu_{A}\left(x_{i}\right)\right] .
$$

$H(A)$ is an entropy measure for fuzzy set $A$. It is easy to get that $H(A)$ is the average of $H_{i}(A)$, for all $x_{i} \in X$.

Proof. (1)

$$
\begin{aligned}
0 \leq & H_{i}(A)=-4 \mu_{A}^{2}\left(x_{i}\right)+4 \mu_{A}\left(x_{i}\right) \leq 1 \Leftrightarrow 0 \leq H(A) \leq 1 \\
& \forall x \in X, \mu_{A}(x) \in\{0,1\}, \\
& H_{i}(A)=-4 \mu_{A}^{2}\left(x_{i}\right)+4 \mu_{A}\left(x_{i}\right)=0 \Leftrightarrow H(A)=0 . \\
\text { (2) } \forall x \in X, \mu_{A}(x)=0.5, & \\
& H_{i}(A)=-4 \times 0.5^{2}+4 \times 0.5=1 \Leftrightarrow H(A)=1 . \\
\text { (3) } \forall x \in X, \mu_{A}(x) \leq \mu_{B}(x) \leq 0.5 . & \\
& \text { According to the monotonicity of function } \\
& f(x)=-4 x^{2}+4 x, \text { we have } \\
& \left\{H_{i}(A)=-4 \mu_{A}^{2}\left(x_{i}\right)+4 \mu_{A}\left(x_{i}\right)\right\} \\
\leq & \left\{H_{i}(B)=-4 \mu_{B}^{2}\left(x_{i}\right)+4 \mu_{B}\left(x_{i}\right)\right\} \Leftrightarrow H(B) \leq H(A) .
\end{aligned}
$$

The same can be obtained:

$$
\begin{aligned}
& \forall x \in X, \mu_{B}(x) \geq \mu_{A}(x) \geq 0.5 \\
& \left\{H_{i}(A)=-4 \mu_{A}^{2}\left(x_{i}\right)+4 \mu_{A}\left(x_{i}\right)\right\} \\
\leq & \left\{H_{i}(B)=-4 \mu_{B}^{2}\left(x_{i}\right)+4 \mu_{B}\left(x_{i}\right)\right\} \Leftrightarrow H(B) \leq H(A) .
\end{aligned}
$$

(4) $\forall A \in F(X), H(A)=H\left(A^{c}\right)$.

$\forall x \in X, \mu_{A^{c}}(x)=1-\mu_{A}(x)$,

$$
\begin{aligned}
H_{i}(A)= & -4\left(\mu_{A}\left(x_{i}\right)-0.5\right)^{2}+1=-4\left[\left(1-\mu_{A}\left(x_{i}\right)\right)-0.5\right]^{2} \\
& +1=H_{i}\left(A^{c}\right) \Leftrightarrow H(A)=H\left(A^{c}\right) .
\end{aligned}
$$

Equation (7) satisfies properties (1)-(4) in Definition 7; thus, $H(A)$ is an entropy measure for fuzzy sets.

\section{Entropy Measures for IFS}

From the definition of intuitionistic fuzzy sets, we can know that the uncertainty of intuitionistic fuzzy sets is jointly determined by the degree of membership, the degree of nonmembership, and the degree of hesitancy. In terms of describing the necessary and sufficient conditions for obtaining the maximum value of intuitionistic fuzzy entropy, Zhang axiomatically states that "when and only when the degrees of membership and nonmembership are both 0.5 , the intuitionistic fuzzy entropy achieves the maximum value". Although the uncertainty of the intuitionistic fuzzy set caused by fuzziness is the largest under this constraint, the hesitancy degree is 0 , and the uncertainty caused by hesitation degree is the smallest. The constraint condition defined by B-B axiomatization describes the necessary and sufficient condition for the maximum value of the intuitionistic fuzzy entropy. It is believed that if and only if the membership degree and nonmembership degree of the element $x$ belonging to $A$ are both 0 , the value of the intuitionistic fuzzy entropy is obtained as maximum value. Contrary to Zhang's axiomatic definition, the hesitancy degree of the intuitionistic fuzzy set is 1 , and the degrees of 


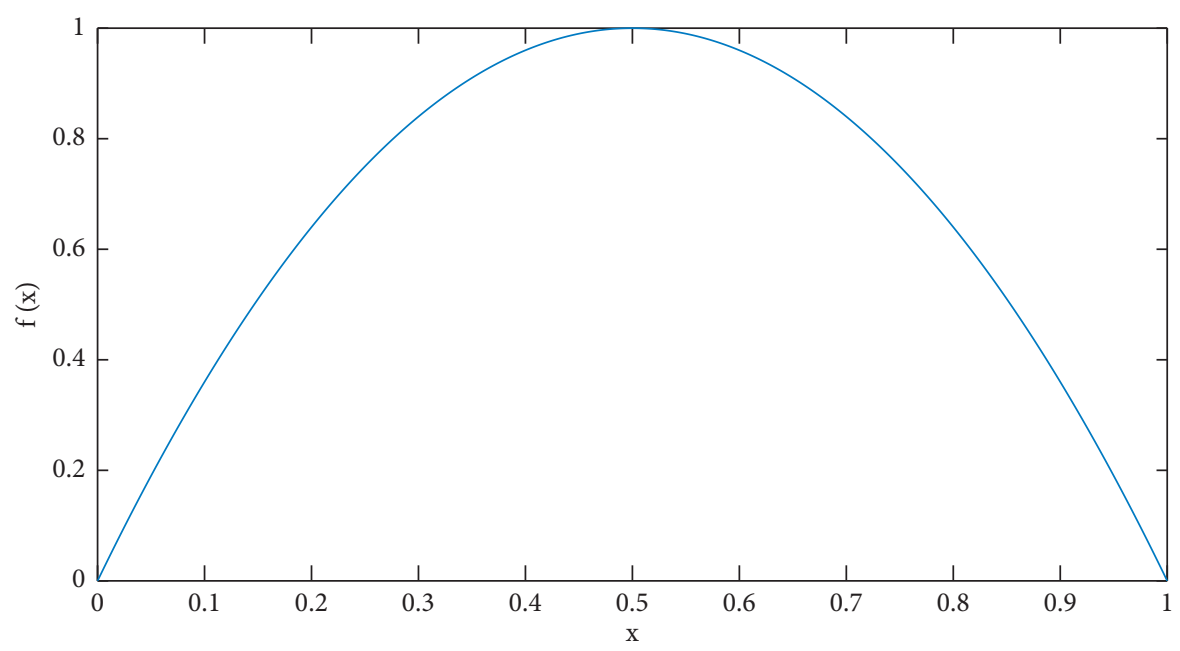

Figure 1: Function $f(x)$.

membership and nonmembership are 0 . The uncertainty caused by hesitancy degree is the largest. The constraint defined by S-K axiomatization is as follows: "when the degree of membership and the degree of nonmembership are equal, the intuitionistic fuzzy entropy reaches its maximum value." This constraint ignores the contribution of the hesitancy degree to intuitionistic fuzzy entropy. When the degrees of membership and nonmembership increase at the same time, the hesitancy degree decreases, and its entropy will decrease accordingly. Therefore, the constraint in the S-K axiomatic definition is contradictory to intuition. With the deepening of research, analyzing the knowledge transferred by Atanassov intuitionistic fuzzy set (AIFS) provides a new perspective for the research of intuitionistic fuzzy entropy. References [21-23] study how to measure the knowledge of intuitionistic fuzzy entropy and propose axiomatic definitions. Reference [24] pointed out that the uncertainty of intuitionistic fuzzy entropy consists of three uncertainties, namely, nonspecificity, fuzziness, and intuitionism. Furthermore, a general uncertainty measure consisting of these three uncertainties is presented.

From the above analysis, the constraints given by each axiomatic definition still have defects, and there is still no unity of the sufficient conditions for the maximum value of intuitionistic fuzzy entropy. In view of this, considering the internal relationship of the three interacting factors of membership degree, nonmembership degree, and hesitancy degree, the axiomatic definition of intuitionistic fuzzy entropy is improved by improving the intuition constraint conditions, and a new definition of intuitionistic fuzzy entropy is proposed accordingly.

The uncertainty of intuitionistic fuzzy sets comes from two aspects. One is the uncertainty of the intuitionistic fuzzy set itself, that is, the uncertainty caused by the fuzziness of membership and nonmembership, and the other is the uncertainty caused by hesitancy degree. Therefore, the construction formula of intuitionistic fuzzy entropy must not only reflect the fuzziness of intuitionistic fuzzy sets, but also reflect the uncertainty of the hesitancy degree. Only by portraying the uncertainty of intuitionistic fuzzy sets from different sides, can the degree of fuzzy intuitionistic fuzzy sets be measured more comprehensively.

3.1. Improved Axiomatic Definition of Intuitionistic Fuzzy Entropy. In order to improve the constraint conditions of the axiomatic definition of intuitionistic fuzzy entropy, the following improved axiomatic definition of intuitionistic fuzzy entropy is proposed.

Definition 10. Let IFSs $(X)$ denote the set of all intuitionistic fuzzy sets over $X$. A mapping $E: \operatorname{IFSs}(X) \longrightarrow[0,1]$ is called an intuitionistic fuzzy entropy if it satisfies the following properties:

(1) $E(A)=0 \Leftrightarrow A$ is a crisp subset

(2) $E(A)=1 \Leftrightarrow \mu_{A}(x)=\nu_{A}(x)=0$ $\mu_{A}(x)=v_{A}(x)=0.5, \forall x \in X$

(3) $E(A)=E\left(A^{c}\right)$

(4) When $\pi_{A}(x)=\pi_{B}(x), \quad\left|\mu_{A}(x)-v_{A}(x)\right| \geq \mid \mu_{B}(x)-$ $\nu_{B}(x) \mid, \forall x \in X, E(A) \leq E(B)$, or when $\left|\mu_{A}(x)-0.5\right|^{2}$ $+\left|v_{A}(x)-0.5\right|^{2}=\left|\mu_{B}(x)-0.5\right|^{2}+\left|v_{B}(x)-0.5\right|^{2}, \pi_{A}$ $(x) \leq \pi_{B}(x), \forall x \in X, E(A) \leq E(B)$

The advantage analysis of the improved axiomatic definition of intuitionistic fuzzy entropy is as follows:

(1) The necessary and sufficient condition for obtaining the minimum value of intuitionistic fuzzy entropy is that the intuitionistic fuzzy set is a crisp subset.

(2) The necessary and sufficient condition for obtaining the maximum value of intuitionistic fuzzy entropy is the degree of membership. When the degree of nonmembership is 0 or 0.5 and when the degrees of membership and nonmembership are 0 , the uncertainty brought by the hesitancy degree is the largest. When the degrees of membership and nonmembership are both 0.5 , the uncertainty caused by the fuzziness is the largest. 
(3) This shows that the entropy of the intuitionistic fuzzy set and that of the complement set are equal.

(4) This constraint states that when the hesitancy degree is equal, the smaller the $\left|\mu_{A}(x)-v_{A}(x)\right|$, the smaller the element $x$ and the greater the uncertainty of the relationship between the set $A$, the greater the intuitionistic fuzzy entropy under this constraint, or when $\left|\mu_{A}(x)-0.5\right|^{2}+\left|\nu_{A}(x)-0.5\right|^{2}=\left|\mu_{B}(x)-0.5\right|^{2}+$ $\left|\nu_{B}(x)-0.5\right|^{2}$, the uncertainty brought by the fuzziness is equal. As the hesitancy degree increases, the intuitionistic fuzzy entropy also increases.

3.2. Intuitionistic Fuzzy Entropy. In order to meet the properties in Definition 10, define $E(A)$ as the entropy of intuitionistic fuzzy.

$$
\begin{aligned}
E(A)= & \frac{1}{n} \sum_{i=1}^{n}\left\{\frac { 1 } { 2 } \left[-4\left(\mu_{A}\left(x_{i}\right)\right)^{2}+4 \mu_{A}\left(x_{i}\right)-4\left(\nu_{A}\left(x_{i}\right)\right)^{2}\right.\right. \\
& \left.\left.+4 \nu_{A}\left(x_{i}\right)\right]+\log _{2}\left(1+\pi^{3}\left(x_{i}\right)\right)\right\} .
\end{aligned}
$$

Among them, $-4\left(\mu_{A}\left(x_{i}\right)\right)^{2}+4 \mu_{A}\left(x_{i}\right)$ describes the contribution of membership to the intuitionistic fuzzy entropy; $-4\left(v_{A}\left(x_{i}\right)\right)^{2}+4 v_{A}\left(x_{i}\right)$ describes the contribution of nonmembership to the intuitionistic fuzzy entropy;

$(1 / 2)\left[-4\left(\mu_{A}\left(x_{i}\right)\right)^{2}+4 \mu_{A}\left(x_{i}\right)-4\left(\nu_{A}\left(x_{i}\right)\right)^{2}+4 v_{A}\left(x_{i}\right)\right]$ describes the uncertainty caused by the fuzziness of membership and nonmembership, and $\log _{2}\left(1+\pi^{3}\left(x_{i}\right)\right)$ describes the uncertainty caused by hesitancy degree. This formula describes the uncertainty of intuitionistic fuzzy sets from the two aspects of membership degree and hesitancy degree and more comprehensively measures the uncertainty of intuitionistic fuzzy sets. The intuitionistic fuzzy entropy is shown in Figure 2.

Proof. Consider the following function:

$$
\begin{aligned}
g(x, y)= & \frac{1}{2}\left[-4 x^{2}+4 x-4 y^{2}+4 y\right]+\log _{2}\left[1+(1-x-y)^{3}\right] \\
& \left\{\begin{array}{l}
0 \leq x \leq 1 \\
0 \leq y \leq 1 \\
0 \leq x+y \leq 1
\end{array}\right.
\end{aligned}
$$

$(1 / 2)\left[-4 x^{2}+4 x-4 y^{2}+4 y\right]=-4(x-0.5)^{2}+1-4(y-$ $0.5)^{2}+1 \geq 0, \log _{2}\left[1+(1-x-y)^{3}\right] \geq 0$, so $g(x, y) \geq 0$,

$$
\left\{\begin{array}{l}
\frac{\partial g}{\partial x}=-4 x+2+\frac{-3(1-x-y)^{2}}{\left[1+(1-x-y)^{3}\right] \cdot \ln 2}=0 \\
\frac{\partial g}{\partial y}=-4 y+2+\frac{-3(1-x-y)^{2}}{\left[1+(1-x-y)^{3}\right] \cdot \ln 2}=0
\end{array}\right.
$$

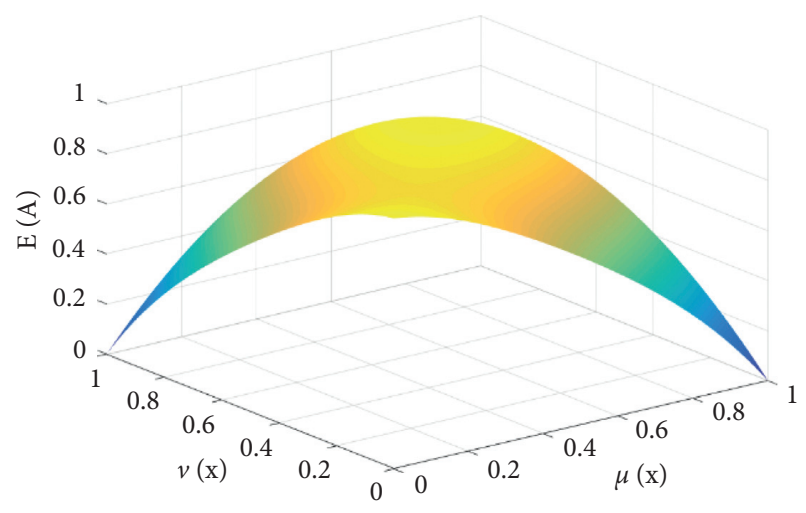

FIgUre 2: Intuitionistic fuzzy entropy $E(A)$.

We can get the stagnation points $(0.2343,0.2343)$ and $(0.5,0.5)$.

Then, we have $g(0.2343,0.2343)=0.9193 \leq 1, g(0.5$, $0.5)=1 \leq 1$.

Consider the boundary value
$-2 x^{2}+2 x+\log _{2}\left[1+(1-x)^{3}\right]$,$\left\{\begin{array}{l}0 \leq x \leq 1 \\ y=0\end{array}, g(x, 0)\right.$

$(\mathrm{d} g / \mathrm{d} x)=-4 x+2+\left(\left(-3(1-x)^{2}\right) /\left(\left[1+(1-x)^{3}\right] \cdot \ln \right.\right.$ $2))=0$. There is no real solution when $0 \leq x \leq 1$, so $(\mathrm{d} g / \mathrm{d} x)<0, g_{\max }(x, y)=g(0,0)=1$.

When $\left.\left\{\begin{array}{l}0 \leq y \leq 1 \\ x=0\end{array}, g\right)^{3}\right], \quad g(0, y)=-2 y^{2}+2 y+\log _{2}[1+(1$

$(\mathrm{d} g / \mathrm{dy})=-4 y+2+\left(\left(-3(1-y)^{2}\right) /\left(\left[1+(1-y)^{3}\right] \cdot \ln \right.\right.$ $2))=0$. There is no real solution when $0 \leq y \leq 1$, so $(\mathrm{d} g / \mathrm{d} x)<0, g_{\max }(x, y)=g(0,0)=1$.

When $\left\{\begin{array}{l}0 \leq x \leq 1 \\ 0 \leq y \leq 1 \\ x+y=1\end{array}, g(x, y)=-2 x^{2}+2 x-2 y^{2}+2 y=g\right.$ $(x, 1-x)=-4 x^{2}+4 x$,

$g_{\max }(x, y)=g(0.5,0.5)=1$, so $g(x, y) \leq 1$.

In summary, $0 \leq g(x, y) \leq 1$.

If $A$ is a crisp subset, we have $A=\{\langle x, 1,0\rangle \mid x \in X\}$ or $A=\{\langle x, 0,1\rangle \mid x \in X\}$.

$$
\begin{aligned}
E(A)= & \frac{1}{n} \sum_{i=1}^{n}\left\{\frac { 1 } { 2 } \left[-4\left(\mu_{A}\left(x_{i}\right)\right)^{2}+4 \mu_{A}\left(x_{i}\right)-4\left(\nu_{A}\left(x_{i}\right)\right)^{2}\right.\right. \\
& \left.\left.+4 v_{A}\left(x_{i}\right)\right]+\log _{2}\left(1+\pi^{3}\left(x_{i}\right)\right)\right\}=0 .
\end{aligned}
$$

$$
\text { If } E(A)=0 \text {, }
$$

$$
\left\{\begin{array}{l}
-4\left(\mu_{A}\left(x_{i}\right)\right)^{2}+4 \mu_{A}\left(x_{i}\right)=0 \\
-4\left(v_{A}\left(x_{i}\right)\right)^{2}+4 v_{A}\left(x_{i}\right)=0 \\
\log _{2}\left(1+\pi^{3}\left(x_{i}\right)\right)=0
\end{array}\right.
$$

$\mid x \in X\}$.

We can get $A=\{\langle x, 1,0\rangle \mid x \in X\}$ or $A=\{\langle x, 0,1\rangle$

If $E(A)=1$, then we have 


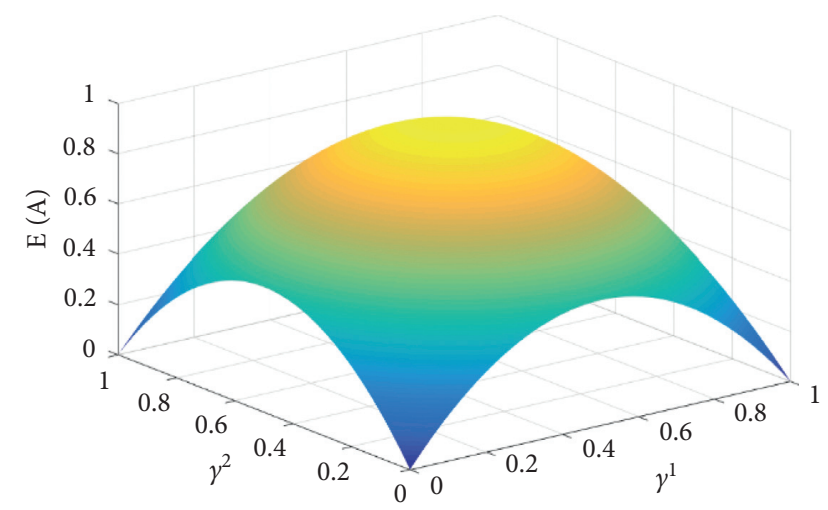

Figure 3: Hesitant fuzzy entropy $E_{i}(A)$. or $\in X\}$.

Therefore, $A=\{\langle x, 0,0\rangle \mid x \in X\}$ or $A=\{\langle x, 0.5,0.5\rangle \mid x$ 
When $\pi_{A}(x)=0$, intuitionistic fuzzy sets degenerate into fuzzy sets. We can get

$$
\begin{aligned}
E(A)= & \frac{1}{n} \sum_{i=1}^{n}\left\{\frac { 1 } { 2 } \left[-4\left(\mu_{A}\left(x_{i}\right)\right)^{2}+4 \mu_{A}\left(x_{i}\right)-4\left(\nu_{A}\left(x_{i}\right)\right)^{2}\right.\right. \\
& \left.\left.+4 v_{A}\left(x_{i}\right)\right]\right\}=0 .
\end{aligned}
$$

Since $\pi_{A}(x)=0, \mu_{A}(x)=1-v_{A}(x)$; then, we have $E(A)=(1 / n) \sum_{i=1}^{n}\left\{\left[-4\left(\mu_{A}\left(x_{i}\right)\right)^{2}+4 \mu_{A}\left(x_{i}\right)\right]\right\}$.

It is easy to get that intuitionistic fuzzy entropy degenerates into fuzzy entropy.

\section{Conclusion}

This paper proposes new fuzzy entropy based on the axiomatic definition of fuzzy entropy, and on this basis, the paper analyzes the existing problems in the axiomatic definitions of intuitionistic fuzzy entropy and hesitant fuzzy entropy and proposes improvements.

Improvements to the new intuitionistic fuzzy entropy are as follows:

(1) We improve the conditions for taking the maximum value of the intuitionistic fuzzy entropy and propose that when the degree of blur or hesitancy of the intuitionistic fuzzy entropy is the maximum, the intuitionistic fuzzy entropy is the maximum value

(2) Intuitionistic fuzzy entropy is a real-valued continuous function increasing with the hesitancy degree and decreasing with $\left|\mu_{A}(x)-\nu_{A}(x)\right|$

Improvements to the new hesitant fuzzy entropy are as follows:

(1) Aiming at the situation where the hesitant fuzzy entropy takes the maximum value, we propose that only when all the elements in the hesitant fuzzy element are 0.5 , does the hesitant fuzzy entropy obtain the maximum value

(2) Aiming at the comparison and improvement of the hesitant fuzzy entropy, we propose that only when the lengths of the hesitant fuzzy elements are equal, is a method for judging the hesitant fuzzy entropy proposed when the lengths of the HFSs are not equal

Through theoretical verification, the new intuitionistic fuzzy entropy and hesitant fuzzy entropy conform to the axiomatic definition and are more effective than the previous intuitionistic fuzzy entropy and hesitant fuzzy entropy. Moreover, this paper unifies the fuzzy entropy, intuitionistic fuzzy entropy, and hesitant fuzzy entropy in form, so that when the intuitionistic fuzzy set and hesitant fuzzy set degenerate into ordinary fuzzy sets, the intuitionistic fuzzy entropy and hesitant fuzzy entropy also degenerate into fuzzy entropy.

\section{Appendix}

The specific certification process is as follows. Accordingly, we have $E(A) \leq E(B), \forall x \in X$.

\section{A. Entropy Measures for HFSs}

In hesitant fuzzy environment, Xu and Xia [19] proposed an entropy axiomatic definition and some formulas for hesitant fuzzy elements (HFEs).

Definition A.1. (see [19]). An entropy on HFE $\alpha$ is a realvalued function $\mathrm{E}: \mathrm{H} \longrightarrow[0,1]$, satisfying the following axiomatic requirements:

(1) $E(\alpha)=0$, if and only if $\alpha=0$ or $\alpha=1$

(2) $E(\alpha)=1$, if and only if $\alpha_{\sigma(i)}+\alpha_{\sigma(l-i+1)}=1$, for $i=1,2, \ldots, l_{\alpha}$

(3) $E(\alpha) \leq E(\beta)$, if $\alpha_{\sigma(i)} \leq \beta_{\sigma(i)}$ for $\beta_{\sigma(i)}+\beta_{\sigma(l-i+1)} \leq 1$ or $\alpha_{\sigma(i)} \geq \beta_{\sigma(i)}$, for $\beta_{\sigma(i)}+\beta_{\sigma(l-i+1)} \geq 1, i=1,2, \ldots, l$

(4) $E(\alpha)=E\left(\alpha^{c}\right)$

It can be seen from Definition a.1 that for HFE $h=\{0.4,0.5,0.6\}$, its hesitant fuzzy entropy is 1 . According to Definition a.1, the fuzzy entropy of element 0.5 is 1 , and the fuzzy entropy of elements 0.4 and 0.6 should be equal and less than 1 . Therefore, the hesitant fuzzy entropy of the hesitant fuzzy element $h$ should also be less than 1 . Therefore, it is not difficult to see that (2) in Definition a.1 needs to be further improved, and the constraint of (3) that the number of elements in the two HFEs must be equal is too high. For two hesitant fuzzy elements with unequal length, it is impossible to judge their entropy value.

In order to perfect the constraint conditions of the axiomatic definition of hesitant fuzzy entropy, a new axiomatic definition of hesitant fuzzy entropy will be given below.

\section{B. Improved Axiomatic Definition of Hesitant Fuzzy Entropy}

Definition B.1. Suppose a domain $X=\left\{x_{1}, x_{2}, \ldots, x_{n}\right\}, A=$ $\left\{\left\langle x_{i}, h_{A}\left(x_{i}\right)\right\rangle \mid x_{i} \in X\right\}$ is a HFS on $X$, and there is a HFE $h_{A}\left(x_{i}\right)=\left\{\gamma_{i}^{1}, \gamma_{i}^{2}, \ldots, \gamma_{i}^{l_{i}}\right\}$, where $l_{i}$ is the number of values in $h_{A}$ and is called the length of $h_{A}$. If the mapping $E: \operatorname{HFS}(X) \longrightarrow[0,1]$ satisfies the following four properties, then $E$ is called the entropy of the HFS $A$ :

(1) $E(A)=0$ if and only if $\gamma_{i}^{\lambda}=0$ or $\gamma_{i}^{\lambda}=1$, $i=1,2, \ldots, n, \lambda=1,2, \ldots, l_{i}$

(2) $E(A)=1$ if and only if $A$ is the fuzziest set; that is, $\gamma_{i}^{\lambda}=0.5, i=1,2, \ldots, n, \lambda=1,2, \ldots, l_{i}$

(3) $E(A)=E\left(A^{c}\right)$, where $A^{c}$ is the complement of $A$

(4) When $l_{A}=l_{B}$, if $\sum_{\lambda=1}^{l_{A}}\left|\gamma_{i}^{\lambda}-0.5\right|^{2} \leq \sum_{\lambda=1}^{l_{B}}\left|\gamma_{i}^{\lambda}-0.5\right|^{2}$, $\forall x \in X, \quad E(A) \geq E(B)$, or when $\sum_{\lambda=1}^{\mathcal{I}_{A}^{\lambda}}\left|\gamma_{i}^{\lambda}-0.5\right|^{2}=$ $\sum_{\lambda=1}^{l_{B}}\left|\gamma_{i}^{\lambda}-0.5\right|^{2}$, if $l_{A} \leq l_{B}, \forall x \in X, E(A) \geq E(B)$ 
The advantage analysis of the improved axiomatic definition of hesitant fuzzy entropy is as follows:

(1) Property (2) shows the necessary and sufficient conditions for the hesitant fuzzy entropy to obtain the maximum value. Only when the elements in the hesitation fuzzy element are all 0.5 , does the hesitant fuzzy entropy take the maximum value. This property improves the condition for obtaining the maximum value of the hesitant fuzzy entropy, which is more in line with reality.

(2) Property (4) provides a method for comparing the entropy of hesitating fuzzy elements when the lengths of the hesitation fuzzy elements are equal or not equal.

\section{Hesitant Fuzzy Entropy}

Before defining the hesitant fuzzy entropy of the HFS, we first define the hesitant fuzzy entropy of the HFE. In order to meet the properties in Definition b.1, define $E_{i}(A)$ as the entropy of HFE.

$$
E_{i}(A)=\frac{1}{l_{i}} \sum_{\lambda=1}^{l_{i}}\left[-4\left(\gamma_{i}^{\lambda}\right)^{2}+4 \gamma_{i}^{\lambda}\right]
$$

Since $f(x)=-4 x^{2}+4 x$, for $0 \leq x \leq 1$, we can get $0 \leq E_{i}(A) \leq 1$.

When the HFE has only two elements, the hesitant fuzzy entropy is shown in Figure 3.

Proof. If $\gamma_{i}^{\lambda}=0$ or $\gamma_{i}^{\lambda}=1, i=1,2, \ldots, n, \lambda=1,2, \ldots, l_{i}$, we can get $E_{i}(A)=\left(1 / l_{i}\right) \sum_{\lambda=1}^{l_{i}}\left[-4\left(\gamma_{i}^{\lambda}\right)^{2}+4 \gamma_{i}^{\lambda}\right]=0$.

If $E_{i}(A)=\left(1 / l_{i}\right) \sum_{\lambda=1}^{l_{i}}\left[-4\left(\gamma_{i}^{\lambda}\right)^{2}+4 \gamma_{i}^{\lambda}\right]=0$, it is easy to get that $-4\left(\gamma_{i}^{\lambda}\right)^{2}+4 \gamma_{i}^{\lambda}=0$.

Therefore, $\gamma_{i}^{\lambda}=0$ or 1 .

If $A$ is the fuzziest set, we have $\gamma_{i}^{\lambda}=0.5, i=1,2, \ldots, n$, $\lambda=1,2, \ldots, l_{i}$.

Then, we can get $E_{i}(A)=\left(1 / l_{i}\right) \sum_{\lambda=1}^{l_{i}}\left[-4\left(\gamma_{i}^{\lambda}\right)^{2}+4 \gamma_{i}^{\lambda}\right]=1$.

If $E_{i}(A)=\left(1 / l_{i}\right) \sum_{\lambda=1}^{l_{i}}\left[-4\left(\gamma_{i}^{\lambda}\right)^{2}+4 \gamma_{i}^{\lambda}\right]=1$, we can get $-4\left(\gamma_{i}^{\lambda}\right)^{2}+4 \gamma_{i}^{\lambda}=1$; therefore, $\gamma_{i}^{\lambda}=0.5$.

$$
\begin{aligned}
E_{i}(A) & =\frac{1}{l_{i}} \sum_{\lambda=1}^{l_{i}}\left[-4\left(\gamma_{i}^{\lambda}\right)^{2}+4 \gamma_{i}^{\lambda}\right] \\
& =\frac{1}{l_{i}} \sum_{\lambda=1}^{l_{i}}\left[-4\left(1-\gamma_{i}^{\lambda}\right)^{2}+4\left(1-\gamma_{i}^{\lambda}\right)\right]=E_{i}\left(A^{c}\right) .
\end{aligned}
$$

It is easy to get that $E(A)=E\left(A^{c}\right)$.

When $\quad l_{A}=l_{B}$, if $\quad \sum_{\lambda=1}^{l_{A}}\left|\gamma_{i}^{\lambda}-0.5\right|^{2} \leq \sum_{\lambda=1}^{l_{B}}\left|\gamma_{i}^{\lambda}-0.5\right|^{2}$, $\forall x \in X$,

$$
\begin{aligned}
& E_{i}(A)=\frac{1}{l_{A}} \sum_{\lambda=1}^{l_{A}}\left[-4\left(\gamma_{i}^{\lambda}\right)^{2}+4 \gamma_{i}^{\lambda}\right]=\frac{1}{l_{A}} \sum_{\lambda=1}^{l_{A}}\left[-4\left(\gamma_{i}^{\lambda}-0.5\right)^{2}+1\right] \geq \\
& E_{i}(B)=\frac{1}{l_{B}} \sum_{\lambda=1}^{l_{B}}\left[-4\left(\gamma_{i}^{\lambda}\right)^{2}+4 \gamma_{i}^{\lambda}\right]=\frac{1}{l_{B}} \sum_{\lambda=1}^{l_{B}}\left[-4\left(\gamma_{i}^{\lambda}-0.5\right)^{2}+1\right] \Rightarrow E(A) \geq E(B) .
\end{aligned}
$$

When $\quad \sum_{\lambda=1}^{l_{A}}\left|\gamma_{i}^{\lambda}-0.5\right|^{2}=\sum_{\lambda=1}^{l_{B}}\left|\gamma_{i}^{\lambda}-0.5\right|^{2}, \quad$ if $\quad l_{A} \leq l_{B}$, $\forall x \in X$,

$$
\begin{aligned}
& E_{i}(A)=\frac{1}{l_{A}} \sum_{\lambda=1}^{l_{A}}\left[-4\left(\gamma_{i}^{\lambda}\right)^{2}+4 \gamma_{i}^{\lambda}\right]=\frac{1}{l_{A}} \sum_{\lambda=1}^{l_{A}}\left[-4\left(\gamma_{i}^{\lambda}-0.5\right)^{2}+1\right] \geq \\
& E_{i}(B)=\frac{1}{l_{B}} \sum_{\lambda=1}^{l_{B}}\left[-4\left(\gamma_{i}^{\lambda}\right)^{2}+4 \gamma_{i}^{\lambda}\right]=\frac{1}{l_{B}} \sum_{\lambda=1}^{l_{B}}\left[-4\left(\gamma_{i}^{\lambda}-0.5\right)^{2}+1\right] \Rightarrow E_{i}(A) \geq E_{i}(B) .
\end{aligned}
$$

Next, we define the hesitant fuzzy entropy of HFSs.

$$
E(A)=\frac{1}{n} \sum_{i=1}^{n}\left\{-\frac{1}{l_{i}} \sum_{\lambda=1}^{l_{i}}\left[-4\left(\gamma_{i}^{\lambda}\right)^{2}+4 \gamma_{i}^{\lambda}\right]\right\} .
$$

It is easy to get $0 \leq E(A) \leq 1$.

If $\gamma_{i}^{\lambda}=0$ or $\gamma_{i}^{\lambda}=1, \quad i=1,2, \ldots, n, \lambda=1,2, \ldots, l_{i}$, $E(A)=(1 / n) \sum_{i=1}^{n}\left\{\left(1 / l_{i}\right) \sum_{\lambda=1}^{l_{i}}\left[-4\left(\gamma_{i}^{\lambda}\right)^{2}+4 \gamma_{i}^{\lambda}\right]\right\}=0$.
If $E(A)=(1 / n) \sum_{i=1}^{n}\left\{\left(1 / l_{i}\right) \sum_{\lambda=1}^{l_{i}}\left[-4\left(\gamma_{i}^{\lambda}\right)^{2}+4 \gamma_{i}^{\lambda}\right]\right\}=0$, $-4\left(\gamma_{i}^{\lambda}\right)^{2}+4 \gamma_{i}^{\lambda}=0$, so $\gamma_{i}^{\lambda}=0$ or 1 .

If $A$ is the fuzziest set, $\gamma_{j}^{\lambda}=0.5, i=1,2, \ldots, n$, $\lambda=1,2, \ldots, l_{i}, \quad E(A)=(1 / n) \sum_{i=1}^{n}\left\{\left(1 / l_{i}\right) \sum_{\lambda=1}^{l_{i}}\left[-4\left(\gamma_{i}^{\lambda}\right)^{2}+4\right.\right.$ $\left.\left.\gamma_{i}^{\lambda}\right]\right\}=1$.

$$
\text { If } E(A)=(1 / n) \sum_{i=1}^{n}\left\{\left(1 / l_{i}\right) \sum_{\lambda=1}^{l_{i}} \sum_{l_{i}}\left[-4\left(\gamma_{i}^{\lambda}\right)^{2}+4 \gamma_{i}^{\lambda}\right]\right\}=1 \text {, }
$$
$-4\left(\gamma_{i}^{\lambda}\right)^{2}+4 \gamma_{i}^{\lambda}=1$, so $\gamma_{i}^{\lambda}=0.5$ 


$$
\begin{aligned}
E(A) & =\frac{1}{n} \sum_{i=1}^{n}\left\{\frac{1}{l_{i}} \sum_{\lambda=1}^{l_{i}}\left[-4\left(\gamma_{i}^{\lambda}\right)^{2}+4 \gamma_{i}^{\lambda}\right]\right\}=\frac{1}{n} \sum_{i=1}^{n}\left\{-\frac{1}{l_{i}} \sum_{\lambda=1}^{l_{i}}\left[-4\left(1-\gamma_{i}^{\lambda}\right)^{2}+4\left(1-\gamma_{i}^{\lambda}\right)\right]\right\} \\
& =E\left(A^{c}\right) \Leftrightarrow E(A)=E\left(A^{c}\right) .
\end{aligned}
$$

When $\quad l_{A}=l_{B}, \quad$ if $\quad \sum_{\lambda=1}^{l_{A}}\left|\gamma_{i}^{\lambda}-0.5\right|^{2} \leq \sum_{\lambda=1}^{l_{B}}\left|\gamma_{i}^{\lambda}-0.5\right|^{2}$, $\forall x \in X$

$$
\begin{aligned}
& E(A)=\frac{1}{n} \sum_{i=1}^{n}\left\{\frac{1}{l_{A}} \sum_{\lambda=1}^{l_{A}}\left[-4\left(\gamma_{i}^{\lambda}\right)^{2}+4 \gamma_{i}^{\lambda}\right]\right\}=\frac{1}{n} \sum_{i=1}^{n}\left\{\frac{1}{l_{A}} \sum_{\lambda=1}^{l_{A}}\left[-4\left(\gamma_{i}^{\lambda}-0.5\right)^{2}+1\right]\right\} \geq \\
& E(B)=\frac{1}{n} \sum_{i=1}^{n}\left\{\frac{1}{l_{B}} \sum_{\lambda=1}^{l_{B}}\left[-4\left(\gamma_{i}^{\lambda}\right)^{2}+4 \gamma_{i}^{\lambda}\right]\right\}=\frac{1}{n} \sum_{i=1}^{n}\left\{\frac{1}{l_{B}} \sum_{\lambda=1}^{l_{B}}\left[-4\left(\gamma_{i}^{\lambda}-0.5\right)^{2}+1\right]\right\} \Rightarrow E(A) \geq E(B) .
\end{aligned}
$$

$\forall x \in X$,

When $\quad \sum_{\lambda=1}^{l_{A}}\left|\gamma_{i}^{\lambda}-0.5\right|^{2}=\sum_{\lambda=1}^{l_{B}}\left|\gamma_{i}^{\lambda}-0.5\right|^{2}, \quad$ if $\quad l_{A} \leq l_{B}$,

$$
\begin{aligned}
& E(A)=\frac{1}{n} \sum_{i=1}^{n}\left\{\frac{1}{l_{A}} \sum_{\lambda=1}^{l_{A}}\left[-4\left(\gamma_{i}^{\lambda}\right)^{2}+4 \gamma_{i}^{\lambda}\right]\right\}=\frac{1}{n} \sum_{i=1}^{n}\left\{\frac{1}{l_{A}} \sum_{\lambda=1}^{l_{A}}\left[-4\left(\gamma_{i}^{\lambda}-0.5\right)^{2}+1\right]\right\} \geq \\
& E(B)=\frac{1}{n} \sum_{i=1}^{n}\left\{\frac{1}{l_{B}} \sum_{\lambda=1}^{l_{B}}\left[-4\left(\gamma_{i}^{\lambda}\right)^{2}+4 \gamma_{i}^{\lambda}\right]\right\}=\frac{1}{n}\left\{\sum_{i=1}^{n} \frac{1}{l_{B}} \sum_{\lambda=1}^{l_{B}}\left[-4\left(\gamma_{i}^{\lambda}-0.5\right)^{2}+1\right]\right\} \Rightarrow E(A) \geq E(B) .
\end{aligned}
$$

From the above discussion, we can get that (11) satisfies conditions (1)-(4) in Definition b.1; thus, $E(A)$ is an entropy measure for HFEs.

If there is only one value in each $h_{A}\left(x_{i}\right)(i=1,2, \ldots, n)$, then the HFS $A$ degenerates into an ordinary fuzzy set. Then, we can get

$$
E(A)=\frac{1}{n} \sum_{i=1}^{n}\left\{\left[-4\left(\gamma_{i}^{\lambda}\right)^{2}+4 \gamma_{i}^{\lambda}\right]\right\}
$$

It is easy to get that hesitant fuzzy entropy degenerates into fuzzy entropy.

\section{Data Availability}

No data were used to support this study.

\section{Conflicts of Interest}

The authors declare that they have no conflicts of interest.

\section{Supplementary Materials}

See the appendix for the specific certification process. Accordingly, we have $E(A) \leq E(B), \forall x \in X$. (Supplementary Materials)

\section{References}

[1] L. A. Zadeh, "Fuzzy sets," Information and Control, vol. 8, no. 3, pp. 338-353, 1965.

[2] A. De Luca and S. Termini, "A definition of a nonprobabilistic entropy in the setting of fuzzy sets theory," Information and Control, vol. 20, no. 4, pp. 301-312, 1972.

[3] K. T. Atanassov, "Intuitionistic fuzzy sets," Fuzzy Sets and Systems, vol. 20, no. 1, pp. 87-96, 1986.

[4] K. T. Atanassov, "More on intuitionistic fuzzy sets," Fuzzy Sets and Systems, vol. 33, no. 1, pp. 37-45, 1989.

[5] K. T. Atanassov, "New operations defined over the intuitionistic fuzzy sets," Fuzzy Sets and Systems, vol. 61, no. 2, pp. 137-142, 1994.

[6] K. Atanassov, On Intuitionistic Fuzzy Sets Theory, Springer, Berlin, Germany, 2012.

[7] DF. Li, "Multiattribute decision making models and methods using intuitionistic fuzzy sets," Journal of Computer and System Sciences, vol. 70, pp. 73-85, 2004.

[8] G. Wang, J. Zhang, and Y. F. Song, "An entropy-based knowledge measure for atanassov's intuitionistic fuzzy sets and its application to multiple attribute decision making," Entropy, vol. 20, no. 12, 2018.

[9] M. Xia and Z. Xu, "Entropy/cross entropy-based group decision making under intuitionistic fuzzy environment," Information Fusion, vol. 13, no. 1, pp. 31-47, 2012.

[10] K. Ioannis and D. George, "Intuitionistic fuzzy information-Applications to pattern recognition," Pattern Recognition Letters, vol. 28, pp. 197-206, 2007. 
[11] D. Kumar, R. K. Agrawal, and H. Verma, "Kernel intuitionistic fuzzy entropy clustering for MRI image segmentation," Soft Computing, vol. 24, no. 6, pp. 4003-4026, 2019.

[12] P. Burillo and H. Bustince, "Entropy on intuitionistic fuzzy sets and on interval-valued fuzzy sets," Fuzzy Sets and Systems, vol. 78, no. 3, pp. 305-316, 1996.

[13] E. Szmidt and J. Kacprzyk, "Entropy for intuitionistic fuzzy sets," Fuzzy Sets and Systems, vol. 118, no. 3, pp. 467-477, 2001.

[14] W.-L. Hung and M.-S. Yang, "Fuzzy entropy on intuitionistic fuzzy sets," International Journal of Intelligent Systems, vol. 21, no. 4, pp. 443-451, 2006.

[15] H. Zhang, W. Zhang, and C. Mei, "Entropy of interval-valued fuzzy sets based on distance and its relationship with similarity measure," Knowledge-Based Systems, vol. 22, no. 6, pp. 449-454, 2009.

[16] V. Torra, "Hesitant fuzzy sets," International Journal of Intelligent Systems, vol. 25, no. 6, pp. 529-539, 2010.

[17] C. Wei, F. Yan, and R. M. Rodríguez, "Entropy measures for hesitant fuzzy sets and their application in multi-criteria decision-making," Journal of Intelligent and Fuzzy Systems, vol. 31, no. 1, pp. 673-685, 2016.

[18] J. Hu, X. Zhang, X. Chen, and Y. Liu, "Hesitant fuzzy information measures and their applications in multi-criteria decision making," International Journal of Systems Science, vol. 47, no. 1, pp. 62-76, 2016.

[19] Z. Xu and M. Xia, "Hesitant fuzzy entropy and cross-entropy and their use in multiattribute decision-making," International Journal of Intelligent Systems, vol. 27, no. 9, pp. 799822, 2012.

[20] W. Liang, M. Goh, and Y. M. Wang, "Multi-attribute group decision making method based on prospect theory under hesitant probabilistic fuzzy environment," Computers \& Industrial Engineering, vol. 149, 2020.

[21] N. R. Pal, H. Bustince, M. Pagola, U. K. Mukherjee, D. P. Goswami, and G. Beliakov, "Uncertainties with Atanassov's intuitionistic fuzzy sets: fuzziness and lack of knowledge," Information Sciences, vol. 228, pp. 61-74, 2013.

[22] E. Szmidt, J. Kacprzyk, and P. Bujnowski, "How to measure the amount of knowledge conveyed by Atanassov's intuitionistic fuzzy sets," Information Sciences, vol. 257, pp. 276285, 2014.

[23] KH. Guo, "Knowledge measure for Atanassov's intuitionistic fuzzy sets," IEEE Transactions on Fuzzy Systems, vol. 24, no. 5, 2017.

[24] Y. Song, X. Wang, W. Wu, L. Lei, and W. Quan, "Uncertainty measure for Atanassov's intuitionistic fuzzy sets," Applied Intelligence, vol. 46, no. 4, pp. 757-774, 2017. 